\title{
Square-wave adsorptive stripping voltammetric approaches at two in situ modified electrodes as first analytical methods for the quantitative determination of a new anticancer drug candidate
}

\author{
Anna Stępniowska ${ }^{1}$ (1) $\cdot$ Małgorzata Sztanke ${ }^{2} \cdot$ Tomasz Tuzimski $^{3} \cdot$ Mieczysław Korolczuk $^{4} \cdot$ Krzysztof Sztanke $^{5}$
}

Received: 25 January 2019 / Accepted: 12 July 2019 / Published online: 19 July 2019

(c) The Author(s) 2019

\begin{abstract}
The two adsorptive stripping voltammetric approaches for detection and quantitative determination of diethyl (2E)2-\{(2E)-[1-(4-methylphenyl)imidazolidin-2-ylidene]hydrazinylidene $\}$ butanedioate (DIB) - a novel molecule of medical importance-using two sensitive sensors based on modified glassy carbon electrodes as reusable sensors, were developed for the first time. The proposed electrochemical methods are based on adsorptive/reductive behaviour of DIB at two modified carbonic electrodes: a bismuth film-modified glassy carbon electrode (BiF/GCE) and a lead film-modified glassy carbon electrode $(\mathrm{PbF} / \mathrm{GCE})$. The electron gain mechanism for the electrochemical reduction of DIB on both developed sensors was proposed for the first time. To achieve the highest sensitivity in adsorptive stripping determinations, various experimental variables (e.g. the composition and $\mathrm{pH}$ of the supporting electrolytes, deposition conditions of bismuth and lead films, concentrations of plating solutions, accumulation times and potentials of DIB, etc.) were extensively examined. The comparison of validation parameters obtained during the determination of DIB at two sensors was presented. The excellent linear correlation was found between the monitored adsorptive stripping voltammetric peak current and the DIB concentration in the range of 15-600 $\mu \mathrm{g} \mathrm{L}^{-1}$ at an accumulation time of $30 \mathrm{~s}$ (with $\mathrm{LOD}=4.2 \mu \mathrm{g} \mathrm{L}^{-1}$ and $\mathrm{LOQ}=14.0 \mu \mathrm{g} \mathrm{L}^{-1}$ ) using the BiF/GCE as a sensor. Furthermore, the excellent linear relationship was confirmed between the monitored adsorptive stripping voltammetric peak current and the DIB concentration in the range of 9-900 $\mu \mathrm{g} \mathrm{L}^{-1}$ at an accumulation time of $10 \mathrm{~s}$ (with better $\mathrm{LOD}=1.5 \mu \mathrm{g} \mathrm{L}{ }^{-1}$ and $\mathrm{LOQ}=5.0 \mu \mathrm{g} \mathrm{L}^{-1}$ ), employing the $\mathrm{PbF} / \mathrm{GCE}$ as a sensor. The two optimized adsorptive stripping voltammetric approaches-as facile, sensitive, reliable and inexpensive-were successfully used as first methods for the quantitative analysis of a novel anticancer agent (DIB) in its pure pharmaceutically acceptable form. However, the practical applicability of square-wave adsorptive stripping voltammetric determination of the electroactive DIB molecule at a $\mathrm{PbF} / \mathrm{GCE}$, as the modified electrode of higher sensitivity, was presented after its successful solid phase extraction from a real serum sample.
\end{abstract}

Keywords Bismuth film-modified glassy carbon electrode $\cdot$ Lead film-modified glassy carbon electrode $\cdot$ Square-wave adsorptive stripping voltammetry $\cdot$ Potential anticancer drug candidate $\cdot$ Serum sample electroanalysis after SPE

\section{Introduction}

Electronic supplementary material The online version of this article (https://doi.org/10.1007/s13738-019-01735-x) contains supplementary material, which is available to authorized users.

Anna Stępniowska

anna.stepniowska@up.lublin.pl

Extended author information available on the last page of the article
A process for the preparation and applicability of diethyl (2E)-2-\{(2E)-[1-(4-methylphenyl)imidazolidin-2-ylidene] hydrazinylidene butanedioate (DIB) (CAS number 1485685-53-7) (Fig. 1) has been patented previously. Notwithstanding, DIB in its pure pharmaceutically acceptable form is of great significance in relation to the patented medical use. It is worth noting that this novel molecule with prospective medical utility is particularly useful in the treatment of human breast cancer and human multiple myelomas (including those resistant and susceptible to the clinically 


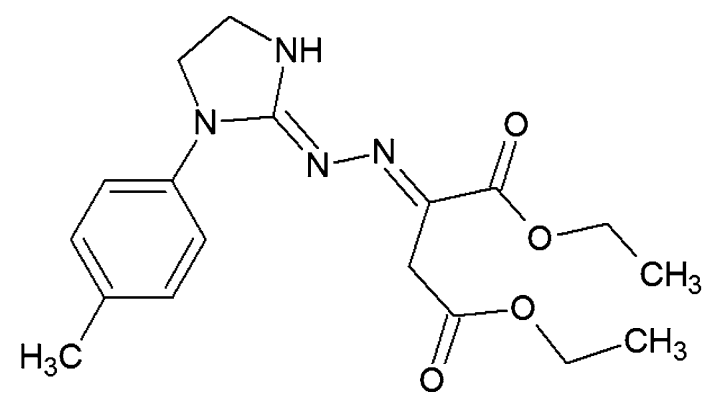

Fig. 1 Structure of diethyl (2E)-2-\{(2E)-[1-(4-methylphenyl)imidazolidin-2-ylidene]hydrazinylidene $\}$ butanedioate (DIB) molecule

approved thalidomide). DIB was identified as the most promising small molecular weight structure in the class of novel imidazolidine-based hybrids with diethyl butanedioate, which may be used in future as an anticancer drug [1,2].

Bioorganic compounds of medical relevance, including approved molecular pharmaceutics, can be quantitatively determined by many methods, mostly HPLC [3], LC-MS [4], GC-MS [5] or stripping voltammetry [6-9]. Adsorptive stripping voltammetry (employing various in situ modified electrodes as sensors) is applied exclusively for the quantitative determination of important bioorganic molecules and pharmaceutics that reveal the ability of adsorptive accumulation at the surface of the working electrode as well as the reductive and/or oxidative behaviour during the analytical stripping step. The main advantages of adsorptive stripping voltammetric analyses are their high selectivity and sensitivity in relation to direct electrochemical methods as well as inexpensiveness (e.g. a low cost of measuring equipment and the decreased intake of organic solvents and production of wastes). The quantitative stripping voltammetric determinations at mercury drop-based electrodes-as sensors-are the most sensitive and selective [10]. Nevertheless, due to the toxicity of liquid mercury, the stripping voltammetric measurements are often carried out on electrodes based on carbon materials, including the modified carbonic electrodes [11-17]. The most popular working electrodes used for determination of electroactive organic compounds, including molecular pharmaceutics, are the bismuth film-modified glassy carbon electrode (BiF/GCE) and lead film-modified glassy carbon electrode ( $\mathrm{PbF} / \mathrm{GCE})$, having in situ deposited bismuth and lead film, respectively, on a glassy carbon support $[6,7,9,11,18-22]$. These modified sensors are relatively simple to prepare, give well-defined electrochemical signals and are less toxic and more environmentally friendly than mercury-based electrodes [9, 22].

Because DIB may be useful in future as a new anticancer drug, the main purpose of the present investigation was to develop and optimize two adsorptive stripping voltammetric methods, as relatively straightforward, sensitive and inexpensive first analytical approaches, allowing its quantitative determination. In addition, the subsequent goal was to elaborate the two modified carbonic electrodes, as reusable working sensors for the analytical purposes, and then to utilize the more sensitive sensor for the determination of DIB in real serum samples (after removal of the interfering matrix components) by means of the optimized square-wave adsorptive stripping voltammetric procedure.

To the best of our knowledge, there is a lack of information about any analytical methods for the quantitative determination of DIB. Hence, the two electrochemical approaches, as first analytical procedures suitable for the quantitative determination of DIB, are presented for the first time in this paper. Up to now, no information about the electrochemical behaviour and reduction mechanism of DIB has been reported in the scientific literature. The electrochemical reduction of DIB was possible due to the attendance of electroactive azomethine bonds $(\mathrm{C}=\mathrm{N})$ under conditions where the protonation step/steps to $\mathrm{CH}-\mathrm{NH}$ grouping/groupings in its parent molecule occurs [23]. The two proposed analytical procedures (with all the optimized experimental parameters affecting the square-wave adsorptive stripping response) are based on adsorptive/reductive behaviour of DIB at two modified carbonic electrodes with in situ deposited thin bismuth or lead film (BiF/GCE and PbF/GCE). It should be clearly mentioned that both developed reusable sensors are used in electroanalysis of DIB for the first time.

\section{Experimental}

\section{Synthesis of DIB}

A novel small molecule of medical importance with the completely defined molecular structure-diethyl $(2 E)$ 2-\{(2E)-[1-(4-methylphenyl)imidazolidin-2-ylidene] hydrazinylidene \}butanedioate (DIB) - has been resynthesized for purposes of current research needs by reacting 1-(4-methylphenyl)-2-hydrazinylideneimidazolidine hydroiodide with diethyl acetylenedicarboxylate in the presence of triethylamine in anhydrous methanolic medium at room temperature, as patented and published earlier $[1,2]$.

\section{HPLC procedure}

For the LC analysis of DIB, the Agilent Technologies 1200 HPLC system equipped with a quaternary pump and a Synergi Polar RP $150 \mathrm{~mm} \times 4.6 \mathrm{~mm}$ column, with $4 \mu \mathrm{m}$ particle size (Phenomenex, Torrance, CA, USA), was employed. The column was thermostatted at ambient temperature. A mobile phase consisting of the following three components was used: acetate buffer $\left(\mathrm{pH} 3.5,0.1 \mathrm{~mol} \mathrm{~L}^{-1}\right)$ and $0.025 \mathrm{~mol} \mathrm{~L}^{-1}$ diethylamine (DEA) (component $\mathrm{A}$ ), $\mathrm{H}_{2} \mathrm{O}$ and $0.025 \mathrm{~mol}$ 
$\mathrm{L}^{-1}$ DEA (component $\mathrm{B}$ ) and acetonitrile and $0.025 \mathrm{~mol} \mathrm{~L}^{-1}$ DEA (component C). An isocratic eluent $(20 \%$ A, $30 \%$ B and $50 \% \mathrm{C}$ ) was employed. The mobile phase flow rate was

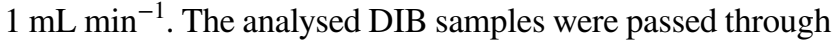
the column. For the manual injection, a Rheodyne valve with a 20- $\mu \mathrm{L}$ loop was used.

\section{Electrodes and instrumentation}

The stripping voltammetric measurements were carried out employing a $\mu$ Autolab analyser combined with a computer (Eco Chemie, the Netherlands). A commercially available glassy carbon serving as the support for the preparation of two modified electrodes- $\mathrm{BiF} / \mathrm{GCE}$ and $\mathrm{PbF} / \mathrm{GCE}-\mathrm{as}$ the working sensors was employed. A conventional threeelectrode glass cell of the $10 \mathrm{~mL}$ volume was used in all electrochemical measurements with an $\mathrm{Ag} / \mathrm{AgCl}$ saturated with $\mathrm{KCl}$ serving as the reference electrode and a platinum wire-as the auxiliary one. The surface of GCE support was polished daily using $0.3 \mu \mathrm{m}$ alumina slurry on a Buehler polishing pad and washed with water. An Elmetron $\mathrm{pH}$ meter CI-316 was employed for $\mathrm{pH}$ measurements. Each measurement to optimize the analytical procedure was carried out at least three times. Before each measurement, the prepared sensors were cleaned electrochemically for their reusability. For this purpose, the lead or bismuth film (deposited on GCE) from the preceding measurement was removed by polarizing the electrode at a potential $1.0 \mathrm{~V}$ for $2 \mathrm{~s}$ two times in the analysed stirred solution.

Solid phase extraction (SPE) of the analysed anticancer agent (DIB) from spiked serum samples was performed using a Baker SPE-12G apparatus (Phillipsburg, USA), equipped with BAKERBOND ${ }^{\text {TM }}$ SPE Octadecyl (C18) cartridges (No. 7020-01, $100 \mathrm{mg} \mathrm{mL}^{-1}$ ) (J.T. Baker, Phillipsburg, USA).

\section{Reagents and solutions}

All the suitable reagents of the analytical grade were purchased from Sigma-Aldrich (Schneldorf, Germany). Exclusively, deionized water was employed during the preparation of all aqueous solutions. A stock $300 \mathrm{mg} \mathrm{L}^{-1}$ methanolic solution of an anticancer agent (DIB) was prepared and stored in the dark until used.

\section{Standard procedure of the stripping voltammetric determinations at a bismuth film-modified glassy carbon electrode (BiF/GCE), as a sensor}

In the optimized measurement conditions, the supporting electrolyte which was employed was acetate buffer $(\mathrm{pH}$ 4.6, $\left.0.02 \mathrm{~mol} \mathrm{~L}^{-1}\right)$. The concentration of $\mathrm{Bi}\left(\mathrm{NO}_{3}\right)_{3}$ added to the electrolyte was $10 \mu \mathrm{mol} \mathrm{L} \mathrm{L}^{-1}$. In our voltammetric measurements, the potential of the electrode changed in the above-mentioned sequence. Firstly, the electrode was cleaned for reuse after the preceding measurement at the potential $1.0 \mathrm{~V}$ for $2 \mathrm{~s}$ two times. Then, a bismuth film was in situ plated on the surface of glassy carbon support at the potential $-1.05 \mathrm{~V}$ for $15 \mathrm{~s}$. During the next step, bismuth(III) was further deposited and DIB was accumulated by adsorption on the surface of $\mathrm{BiF} / \mathrm{GCE}$, as a working sensor, at the potential $-0.65 \mathrm{~V}$ for $30 \mathrm{~s}$. During all these steps, the solution was mixed continuously using a magnetic stirring bar. Then, after a rest period of $5 \mathrm{~s}$, the square-wave adsorptive stripping voltammogram was recorded at a frequency of $100 \mathrm{~Hz}$, while the potential was scanned from -0.6 to $-1.0 \mathrm{~V}$. In turn, the amplitude and step potential were $25 \mathrm{mV}$ and $4.05 \mathrm{mV}$, respectively. The stripping voltammetric measurements were carried out in underaerated solutions as oxygen is reduced on the surface of $\mathrm{BiF} / \mathrm{GCE}$ in one stage at the potential outside a reduction range of this working sensor. Therefore, the presented voltammetric procedure can be employed for determining the DIB in the presence of oxygen on the reduction side. The sensing interface of $\mathrm{BiF} / \mathrm{GCE}$ has been thoroughly characterized by using scanning electron microscopy (SEM) in the previous paper [24].

\section{Standard procedure of the stripping voltammetric determinations at a lead film-modified glassy carbon electrode (PbF/GCE), as a sensor}

In the optimized measurement conditions, the supporting electrolyte which was used was acetate buffer $(\mathrm{pH} 4.0$, $0.05 \mathrm{~mol} \mathrm{~L}^{-1}$ ). The concentration of $\mathrm{Pb}\left(\mathrm{NO}_{3}\right)_{2}$ added to the electrolyte was $50 \mu \mathrm{mol} \mathrm{L}^{-1}$. In our voltammetric measurements, the potential of the electrode changed in the abovementioned sequence. Firstly, the electrode was cleaned for reuse after the preceding measurement at the potential $1.0 \mathrm{~V}$ for $2 \mathrm{~s}$ two times. Next, a lead film was plated on the surface of the electrode at the potential $-1.25 \mathrm{~V}$ for $20 \mathrm{~s}$. Finally, DIB was accumulated by adsorption on the surface of $\mathrm{PbF} /$ GCE, as a working sensor, at the potential $-0.6 \mathrm{~V}$ for $30 \mathrm{~s}$. During all these steps, the solution was stirred continuously employing a magnetic stirring bar. Then, after a rest period of $5 \mathrm{~s}$, the square-wave adsorptive stripping voltammogram was recorded at a frequency of $100 \mathrm{~Hz}$, while the potential was scanned from -0.6 to $-1.1 \mathrm{~V}$. The amplitude and step potential were $25 \mathrm{mV}$ and $4.05 \mathrm{mV}$, respectively. The stripping voltammetric measurements were carried out in underaerated solutions as the reduction of oxygen on the surface of $\mathrm{PbF} / \mathrm{GCE}$ does not form signal in the range of used potentials. The sensing interface of $\mathrm{PbF} / \mathrm{GCE}$ has been carefully characterized by employing SEM technique in previous studies [25-27]. 


\section{Sample preparation and determination of DIB in real serum samples by SW-AdSV at PbF/GCE}

Serum samples $(1 \mathrm{~mL})$ that were spiked with the $5 \mathrm{mg} \mathrm{L}^{-1}$ DIB were incubated at $37{ }^{\circ} \mathrm{C}$ for $60 \mathrm{~min}$. Next, each serum sample was diluted with deionized water $(1 \mathrm{~mL})$. Then, ammonium buffer $\left(0.4 \mathrm{~mL} ; \mathrm{pH}\right.$ 8.6; $\left.0.2 \mathrm{~mol} \mathrm{~L}^{-1}\right)$ was added. Commercially available octadecyl (C18) columns for SPE were activated with methanol $(1 \mathrm{~mL})$ and conditioned with water/ammonium buffer ( $\mathrm{pH} 8.6)$ mixture $(5: 1 ; 1 \mathrm{~mL})$. Then, the serum containing DIB (that was prepared as described above) was passed through the columns (speed $1 \mathrm{~mL} \mathrm{~min}^{-1}$ ). Each column was prewashed with $\mathrm{MeOH} /$ water solution $(35: 65 ; 1 \mathrm{~mL})$ and dried under vacuum conditions for $3 \mathrm{~min}$. The extracted DIB samples were eluted twice with methanol/acetic acid mixture $(49: 1 ; 1 \mathrm{~mL})$, then evaporated to dryness, and finally dissolved in $\mathrm{MeOH}(0.5 \mathrm{~mL})$.

In the next step, DIB was determined using the standard addition method. The square-wave adsorptive stripping voltammetric (SW-AdSV) procedure, using PbF/GCE as a working sensor, was used for the quantitative determination of DIB in real serum samples.

\section{Results and discussion}

\section{Structural analysis of DIB}

The structural characterization $\left({ }^{1} \mathrm{H}\right.$ NMR and ${ }^{13} \mathrm{C}$ NMR spectral data including HMBC, HMQC and elemental analyses) and pharmacological (strong inhibitory effects in human tumour cells) characterization of DIB have been provided in detail previously $[1,2] .{ }^{1} \mathrm{H}$ NMR, ${ }^{13} \mathrm{C}$ NMR and DEPT-135 ${ }^{13} \mathrm{C}$ NMR spectra of DIB are attached in Supplementary material (Figs S1-S3). However, further characterization of DIB by both infrared spectral bands (which was recorded within the spectral range of $4000-500 \mathrm{~cm}^{-1}$ ) with most characteristic vibrational frequencies and characteristic ultraviolet absorption band maxima (with extinction coefficients) of its diluted methanolic solution are presented in this article for the first time.

IR (ATR-FTIR) $\left(\nu, \mathrm{cm}^{-1}\right): 3352$ (NH stretching), 3109, 3087, 3044 (aromatic $\mathrm{CH}$ stretching), 1729 ( $\mathrm{C}=\mathrm{O}$ ester stretching), 1703 ( $\mathrm{C}=\mathrm{O}$ ester stretching), 1603, 1515, 1488 (aromatic skeleton stretching), $1554(\mathrm{C}=\mathrm{N}$ stretching), 1448, 1384 (aromatic $\mathrm{C}-\mathrm{CH}_{3}$ stretching), 815 (1,4-disubstituted benzene ring stretching) UV (50 $\mu \mathrm{M}$ DIB in $\mathrm{MeOH}): \lambda_{\max }$ (ع): $325 \mathrm{~nm}(25,540), \lambda_{\max }{ }^{\prime}(\varepsilon): 244.5 \mathrm{~nm}(11,560)$.

In addition, the analysed anticancer agent (DIB) was detected by comparing-in UV spectra-the registered peaks of DIB reference standards and those derived from serum samples spiked with DIB after SPE.

\section{Adsorptive stripping voltammetric measurements at in situ plated $\mathrm{BiF} / \mathrm{GCE}$ and $\mathrm{PbF} / \mathrm{GCE}$}

\section{Bismuth film-modified glassy carbon electrode (BiF/GCE)}

In our stripping voltammetric determinations of DIB, a glassy carbon was chosen as the support for the first environmentally friendly sensor that was prepared-a bismuth film-modified glassy carbon electrode (BiF/GCE). Main advantages of the usefulness of a commercially available glassy carbon electrode (GCE) support in voltammetric measurements include: a high electrochemical stability, the possibility for use over a wide potential range, a good electrical conductivity and a simplicity of surface renewal $[7$, 28].

Depending on the applied deposition potential, DIB can be reduced in two different ways. On the working sensor-a bismuth film-modified glassy carbon electrode at a deposition potential $-0.7 \mathrm{~V}-\mathrm{a}$ low peak at $-0.71 \mathrm{~V}$ can be observed. While increasing the deposition potential, there appears the second peak at the potential $-0.77 \mathrm{~V}$. In turn, with a deposition potential $-0.4 \mathrm{~V}$, the peak observed at $-0.71 \mathrm{~V}$ disappears, while there is only a signal at $-0.77 \mathrm{~V}$. With the deposition potential $-0.65 \mathrm{~V}$, the highest signal was observed at $-0.71 \mathrm{~V}$, and therefore a further optimization was carried out at this potential (Fig. 2).

The occurrence of two peaks is most probably caused by the double 2e-reduction of two azomethine moieties present in the structure of the analysed compound (DIB). According to this, the electron gain mechanism is also suggested (Fig. $\mathrm{S} 4 \mathrm{~A}$ ). The electrochemical signals of DIB were recorded when the bismuth film-coated GCE was used as a sensor. In turn, there was no electrochemical response in the absence of a bismuth layer on GCE. To check if the reduction steps on the $\mathrm{BiF} / \mathrm{GCE}$ surface are certainly due to two azomethine bonds in DIB molecule, the cyclic voltammogram of a fivemembered heterocyclic compound devoid of any azomethine moieties - tetrahydropyrrole (from Sigma-Aldrich) - was recorded for comparison purposes. In turn, this one did not

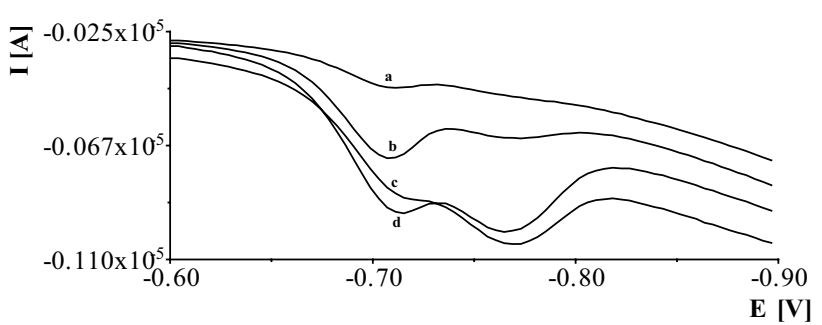

Fig. 2 A square-wave adsorptive stripping voltammogram for $300 \mu \mathrm{g}$ $\mathrm{L}^{-1}$ DIB at the BiF/GCE. DIB was accumulated for $30 \mathrm{~s}$ at $\mathbf{a}-0.7 \mathrm{~V}$, b $-0.65 \mathrm{~V}, \mathbf{c}-0.4 \mathrm{~V}$ and $\mathbf{d}-0.55 \mathrm{~V}$. Concentration of $\mathrm{Bi}\left(\mathrm{NO}_{3}\right)_{3}$ was $10 \mu \mathrm{mol} \mathrm{L}-1$ 
reveal any reduction peaks at potential ranges from -0.6 to $-0.9 \mathrm{~V}$, proving that the two-step 2e-reduction on the surface of that sensor must be caused by two electroactive azomethine bonds in the molecule of DIB.

Further optimization of the important experimental parameters (given in the headings of sections below) affecting the adsorptive stripping response was aimed to achieve a higher sensitivity.

Composition and $\mathrm{pH}$ of the supporting electrolyte The influence of $\mathrm{pH}$ on the height of analyte signal (DIB) was studied over the $\mathrm{pH}$ range 4.0-6.1. It was observed that the highest signal occurs at $\mathrm{pH} 4.6$, and therefore a further optimization was carried out at this $\mathrm{pH}$ value (Fig. 3). Acetate buffer was used to obtain the appropriate $\mathrm{pH}$, and therefore the next parameter tested was the concentration of this buffer. The influence of the concentration of acetate buffer on the signal size in the range from 0.01 to $0.1 \mathrm{~mol} \mathrm{~L}^{-1}$ was thoroughly investigated. The electrochemical signal of DIB was the highest with $0.02 \mathrm{~mol} \mathrm{~L}^{-1}$ acetate buffer and then dropped sharply (Fig. S5). Therefore, a concentration of $0.02 \mathrm{~mol} \mathrm{~L}^{-1}$ was chosen for further measurements.

Optimization of the bismuth film deposition The height of the analyte signal is influenced by the thickness of the bismuth film, which depends on the concentration of bismuth(III) as well as the potential and time of its deposition. The bismuth film was deposited in situ from the mixed solution. At the beginning, the effect of bismuth(III) concentration on the signal size of analyte (DIB) in the range of $2.5-15 \mu \mathrm{mol} \mathrm{L}^{-1}$ was studied. The signal of DIB increased to a concentration of $10 \mu \mathrm{mol} \mathrm{L} \mathrm{L}^{-1}$ and then remained constant, and therefore the concentration of $10 \mu \mathrm{mol} \mathrm{L}^{-1}$ was chosen for a further study (Fig. S6). The deposition potential of bismuth(III) was tested in the range from -1.0 to $-1.2 \mathrm{~V}$. The size of the signal coming from DIB remained stable. The potential $-1.05 \mathrm{~V}$ was selected for further

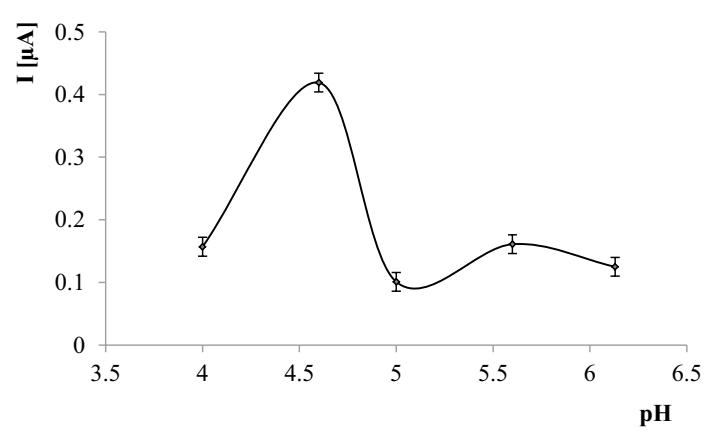

Fig. 3 Effect of the $\mathrm{pH}$ value of the measured solution on the peak current of $300 \mu \mathrm{g} \mathrm{L}^{-1} \mathrm{DIB}$ at the BiF/GCE. The analyte was accumulated for $60 \mathrm{~s}$ at $-0.65 \mathrm{~V}$. Concentration of $\mathrm{Bi}\left(\mathrm{NO}_{3}\right)_{3}$ was $10 \mu \mathrm{mol}$ $\mathrm{L}^{-1}$. Error bars represent the standard deviation $(n=3)$ research. The subsequent parameter that was examined was the deposition time of bismuth(III). Already at $0 \mathrm{~s}$ of deposition time, the analyte was accumulated on the electrode, because at the accumulation potential of DIB the deposition of bismuth(III) proceeds also. However, at a longer accumulation time the electrochemical signal of DIB increased more than twice (Fig. S7). A $15 \mathrm{~s}$ accumulation time was selected as optimal for further studies.

Optimization of an accumulation time of DIB The effect of the accumulation time has been carefully investigated in the range from 0 to $120 \mathrm{~s}$. When increasing the accumulation time to $60 \mathrm{~s}$, the signal height increased. However, a further increase in the accumulation time did not increase the signal height (Fig. S8). Therefore, the accumulation time of $30 \mathrm{~s}$ was selected as optimal for further research.

The calibration graph The calibration graph (Fig. S9) was prepared for a $30 \mathrm{~s}$ accumulation time by plotting the adsorptive stripping peak current (I) in $\mu \mathrm{A}$ against the increasing DIB concentration (c) in $\mathrm{mg} \mathrm{L}^{-1}$. The calibration graph was linear in the range from 0.015 to $0.6 \mathrm{mg} \mathrm{L}^{-1}$ and obeyed the following regression equation: $y=(2.5567 \pm 0.0912)$ $x+(0.0166 \pm 0.0095)$, where $\mathrm{y}$ and $\mathrm{x}$ denote the adsorptive stripping peak current and the analyte (DIB) concentration, respectively (Fig. 4). The linear correlation coefficient was 0.994.

The repeatability of $300 \mu \mathrm{g} \mathrm{L} \mathrm{L}^{-1}$ DIB signals on BiF/GCE expressed as a relative standard deviation was $2.9 \%(n=7)$. The limit of detection (LOD) for peak of the analysed DIB obtained at $\mathrm{BiF} / \mathrm{GCE}$ was calculated as follows: $\mathrm{LOD}=3$ $S D / S$. However, the limit of quantification (LOQ) was calculated as follows: $\mathrm{LOQ}=10 S D / S$, where $S D$ is the standard deviation of y-intercepts $(n=3)$ of regression lines (calculated employing LINES function in MS Excel 2010) and $S$ is the slope of the linear regression equation. The limit of detection of DIB at an accumulation time of $30 \mathrm{~s}$ (using the $\mathrm{BiF} / \mathrm{GCE}$ as a sensor) was found to be $4.2 \mu \mathrm{g} \mathrm{L}^{-1}$, while LOQ was found to be $14.0 \mu \mathrm{g} \mathrm{L}^{-1}$ (Table S2).

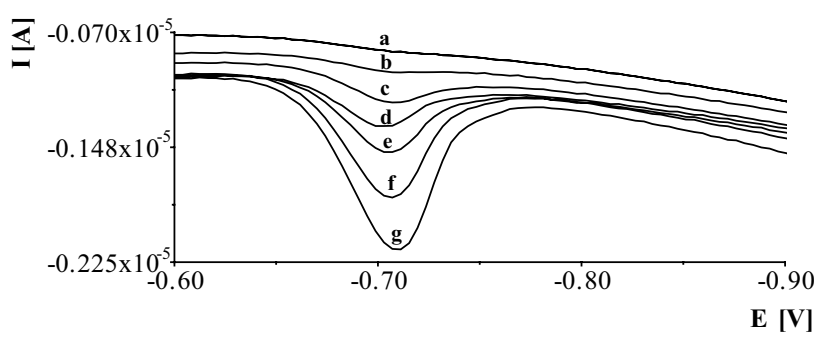

Fig. 4 Square-wave stripping voltammograms obtained for increasing concentrations of DIB at the BiF/GCE: a 0 ; b 0.03; c 0.06; d 0.09 ; e 0.15 ; f 0.3 and $\mathbf{g ~} 0.6 \mathrm{mg} \mathrm{L}^{-1}$, respectively. The analyte was accumulated for $30 \mathrm{~s}$ at $-0.65 \mathrm{~V}$ 


\section{Lead film-modified glassy carbon electrode (PbF/GCE)}

In further stripping voltammetric determinations of DIB, a glassy carbon was again selected as the support for the second prepared sensor-a lead film-modified glassy carbon electrode.

Firstly, we studied the electrochemical response of DIB in the absence as well as attendance of the lead film on a glassy carbon support. The analytical signal of DIB was recorded exclusively when the lead film-coated GCE was used as a sensor. This means that the lead film-modified glassy carbon is necessary to register the DIB electrochemical response. DIB yielded a well-defined single adsorptive stripping voltammetric peak at $-0.72 \mathrm{~V}$ corresponding to the electrochemical reduction of the active $-\mathrm{N}=\mathrm{C}$ bond. Before each measurement, the background with the addition of $\mathrm{Pb}^{2+}\left(50 \mu \mathrm{mol} \mathrm{L}^{-1}\right)$ was recorded and no signal was observed. This means that lead ions cannot interfere with DIB. Our observations showed that the adsorptive stripping voltammetry can be used for the determination of DIB due to its adsorptive/reductive behaviour at the working $\mathrm{PbF} /$ GCE. The occurrence of one reductive peak is most probably caused by the 2e-reduction of only one azomethine moiety in the structure of the analysed compound (DIB) at the working sensor. Based on this, the electron gain mechanism is suggested (Fig. S4B). To prove reliably that the reduction step on the $\mathrm{PbF} / \mathrm{GCE}$ surface is due to one azomethine bond in DIB molecule, a cyclic voltammogram of pyrrolidine (from Sigma-Aldrich)—-the five-membered heterocyclic compound deprived of the azomethine group-was recorded for comparison purposes. This molecule showed no reduction peak at potential ranges from -0.6 to $-0.9 \mathrm{~V}$, proving that the one-step 2e-reduction on the surface of the prepared sensor must be evoked by the electroactive azomethine moiety in the molecule of DIB.

Further optimization of the important experimental variables (provided in the headings of sections below) affecting the adsorptive stripping response was carried out in order to achieve a higher sensitivity.

Composition and $\mathrm{pH}$ of the supporting electrolyte The composition and $\mathrm{pH}$ of the supporting electrolyte have a significant influence on the analyte signal size. The $\mathrm{pH}$ was tested in the range from 3.5 to 6.1. The highest electrochemical signal coming from DIB was observed at $\mathrm{pH}$ 4.0. Further increase in $\mathrm{pH}$ caused a decrease in the signal size, and therefore this $\mathrm{pH}$ value was chosen for further study (Fig. 5). The next parameter tested was the concentration of acetate buffer. Increasing the concentration of acetate buffer to $0.05 \mathrm{~mol} \mathrm{~L}^{-1}$ caused an increase in the signal of DIB, while the use of higher concentrations evoked a gentle decrease in the signal size, and therefore the concentration of $0.05 \mathrm{~mol}$ $\mathrm{L}^{-1}$ was chosen as optimal for further testing (Fig. S10).

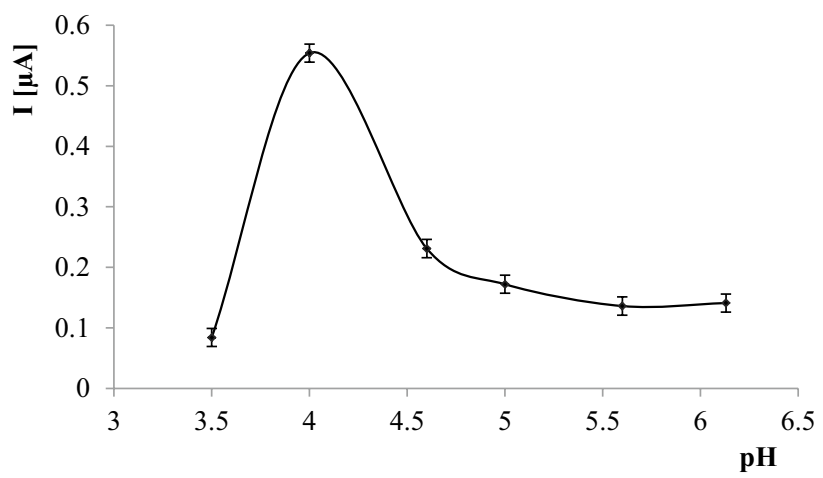

Fig. 5 Effect of the $\mathrm{pH}$ value of the measured solution on the peak current of $300 \mu \mathrm{g} \mathrm{L}^{-1} \mathrm{DIB}$ at the $\mathrm{PbF} / \mathrm{GCE}$. The analyte was accumulated for $30 \mathrm{~s}$ at $-0.6 \mathrm{~V}$. Concentration of $\mathrm{Pb}\left(\mathrm{NO}_{3}\right)_{2}$ was $50 \mu \mathrm{mol}$ $\mathrm{L}^{-1}$. Error bars represent the standard deviation $(n=3)$

Optimization of the lead film deposition The thickness of the lead film depends on the concentration of lead(II) in the supporting electrolyte. In voltammetric measurements, the influence of lead(II) concentration in the range from 2.5 to $100 \mu \mathrm{mol} \mathrm{L}{ }^{-1}$ was examined. The signal from the analysed DIB increases as the concentration of lead(II) increases to $50 \mu \mathrm{mol} \mathrm{L} \mathrm{L}^{-1}$, and then decreases, which is associated with the formation of another layer of lead. A concentration of $50 \mu \mathrm{mol} \mathrm{L}^{-1}$ was selected for a further study (Fig. S11). The influence of lead(II) deposition potential was studied in the range from -1.0 to $-1.35 \mathrm{~V}$. This parameter had a smaller influence on the size of the analytical signal; however, the highest signal was observed at the potential $-1.25 \mathrm{~V}$, and therefore this potential was selected for further research (Fig. S12). The next tested parameter was the deposition time of lead(II). With increasing the time of deposition, the signal coming from DIB increased. For further research, the deposition time of $20 \mathrm{~s}$ was selected, as further elongation of time had a smaller effect on the size of the peak.

Optimization of accumulation time and potential of DIB The next optimized parameter was the accumulation potential of DIB, which was tested in the potential range from -0.55 to $-0.7 \mathrm{~V}$. The highest signal was observed at the potential $-0.6 \mathrm{~V}$, and therefore further investigations were carried out using this accumulation potential (Fig. S13). The size of the analytical signal increased with increasing the accumulation time to $60 \mathrm{~s}$ and then remained constant. A time of $60 \mathrm{~s}$ was selected for further investigations (Fig. S14).

The calibration graph The calibration graphs (Figs S15 and S16) were prepared for two accumulation times of 10 and $60 \mathrm{~s}$ by plotting the adsorptive stripping peak current (I) in $\mu \mathrm{A}$ against the increasing DIB concentration (c) in $\mathrm{mg} \mathrm{L^{-1 }}$. The calibration graph for an accumulation time 
of $10 \mathrm{~s}$ was linear in the range from 0.009 to $0.9 \mathrm{mg} \mathrm{L}^{-1}$ and obeyed the regression equation: $y=(0.5772 \pm 0.0134)$ $x+(0.0226 \pm 0.0134)$, where $y$ and $x$ denote the adsorptive stripping peak current and the DIB concentration, respectively. The linear correlation coefficient was 0.992 . However, the calibration graph for an accumulation time of $60 \mathrm{~s}$ was linear in the range from 0.003 to $0.06 \mathrm{mg} \mathrm{L}^{-1}$ and obeyed the regression equation: $y=(4.764 \pm 0.094)$ $x+(0.0023 \pm 0.0014)\left(R^{2}=0.965\right)$.

The repeatability of $300 \mu \mathrm{g} \mathrm{L} \mathrm{L}^{-1}$ DIB signals on PbF/GCE expressed as a relative standard deviation was $4.1 \%(n=7)$. The limit of detection (LOD) of DIB at an accumulation time of $10 \mathrm{~s}$ (using the $\mathrm{PbF} / \mathrm{GCE}$ as a sensor) was found to be $1.5 \mu \mathrm{g} \mathrm{L}^{-1}$, whereas the limit of quantification (LOQ) was found to be $5.0 \mu \mathrm{g} \mathrm{L}^{-1}$ (Table S2). It should be noted that the analytical sensitivity (which was achieved after a very short accumulation time) seems to be very promising.

\section{Determination of DIB in spiked serum samples at $\mathrm{PbF} /$}

GCE Serum samples as complex matrices are rich in the most abundant proteins, which can interfere in stripping voltammetric measurements. Therefore, the appropriate sample preparation is important. Proteins can be precipitated by trichloroacetic acid, sulfosalicylic acid, perchloric acid or removed via solid phase extraction (SPE). In our studies, the SPE process could be successfully applied in order to remove the interfering proteins in spiked serum samples prior to the quantitative determination of DIB. In turn, the lead film-modified glassy carbon electrode, as a reusable sensor, could be successfully employed for the quantitative determination of DIB. Figure 6 presents the square-wave adsorptive stripping voltammograms obtained for the increasing concentrations of DIB in spiked serum samples using the $\mathrm{PbF} / \mathrm{GCE}$ as a sensor. In order to check the accuracy of the proposed electroanalytical procedure, the standard addition method was employed for the analysis of biological samples spiked with appropriate DIB standards. The mean recovery values are in the accepted range from 92.4 to $107.0 \%$ (Table S1). This

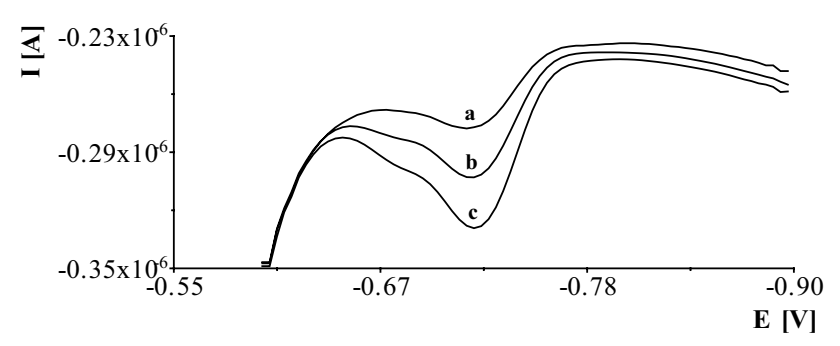

Fig. 6 Square-wave stripping voltammograms obtained for increasing concentrations of DIB in spiked serum samples at the PbF/GCE: a $10 \mu \mathrm{g} \mathrm{L}^{-1}, \mathbf{b}$ as $\mathbf{a}+10 \mu \mathrm{g} \mathrm{L}{ }^{-1} ; \mathbf{c}$ as $\mathbf{a}+20 \mu \mathrm{g} \mathrm{L}^{-1}$. The analyte was accumulated at $-0.6 \mathrm{~V}$ confirmed the satisfactory accuracy and precision of the developed square-wave adsorptive stripping voltammetric procedure for DIB determination in spike serum samples, employing the above-mentioned sensor.

\section{Interferences}

To investigate the interference effects of some organic compounds, a fixed amount of $300 \mu \mathrm{g} \mathrm{L}^{-1}$ DIB spiked with various foreign species, such as glucose, ascorbic acid, glycine, $L$-histidine and $L$-tyrosine, was evaluated under the same experimental conditions. The results proved that a 100-fold excess of these compounds had no influence on the peak current of DIB (changing the analytical signals by less than $5 \%$ ).

\section{Limitations}

The stripping voltammetric determinations of DIB in real serum samples at the $\mathrm{PbF} / \mathrm{GCE}$ as a working sensor must be performed after the SPE-based purification procedure. Moreover, the SPE procedure enhanced the time of sample preparation. The stripping voltammetric determinations of DIB at the BiF/GCE as a sensor should be carried out in acidic and complexing solutions (e.g. acetate buffer $\mathrm{pH}$ 4.6, $0.02 \mathrm{~mol} \mathrm{~L}^{-1}$ according to the Experimental section) due to hydrolysis of bismuth ion (III) under alkaline conditions [29, 30].

\section{Conclusions}

The two optimized adsorptive stripping voltammetric approaches, employing bismuth and lead film-modified glassy carbon electrodes (BiF/GCE and $\mathrm{Pb} / \mathrm{GCE})$, as reusable sensors, were developed as the first analytical procedures allowing the detection and determination of DIB, which may be used in future as an anticancer drug. Both methods proved to be particularly suitable for the quantitative determination of DIB in its pure pharmaceutically accepted form. Extensive and thorough studies were carried out to find the optimal experimental conditions for the quantitative analysis of DIB at two sensors prepared. Furthermore, the square-wave stripping voltammetric procedure using a lead film-modified glassy carbon electrode, as a sensor, proved to be more sensitive, and therefore this one was applied for the successful quantitative analysis of DIB in real serum samples after removing the interfering matrices by SPE. The proposed electroanalytical approaches are based on the adsorptive accumulation of DIB at the surface of working sensors 
and then its reduction ability during the stripping step of electrochemical determination.

When the modified GCE with in situ deposited bismuth film was used as an environmentally friendly sensor in electrochemical measurements, the two peaks at $-0.71 \mathrm{~V}$ and $-0.77 \mathrm{~V}$, connected with the reduction of two azomethine moieties in the structure of DIB, were observed. In turn, when electrochemical determinations were carried out on the lead film-modified GCE as a sensor, the adsorptive stripping voltammetric peak at $-0.72 \mathrm{~V}$, associated with the reduction of only one azomethine moiety in the molecule of DIB, was registered. This can be explained by different reduction behaviours of DIB occurring via electron gain mechanisms at two modified electrodes with in situ deposited bismuth or lead film. Both adsorptive stripping voltammetric procedures, which were developed for the first time, are sensitive enough to determine DIB in its pure form, relatively simple and inexpensive. The main advantage of two electrochemical approaches allowing the quantitative analysis of a new anticancer active molecule is not only the decreased intake of organic solvents, but in addition the lower production of toxic wastes in relation to chromatographic methods. Noteworthy is that the proposed procedures under optimized conditions, using two developed sensors, were successfully applied to the quantitative determination of DIB in its pure pharmaceutically accepted form by means of square-wave adsorptive stripping voltammetry. The surfaces of both modified electrodes were successfully regenerated electrochemically (by using more positive potentials than those in stripping determinations) to remove any deposit on the support from a previous measurement for their reusability.

It should be highlighted that all the interfering components were successfully removed after solid phase extraction from a serum matrix containing DIB. Thereafter, the more sensitive square-wave adsorptive stripping voltammetric approach using a lead film-modified glassy carbon electrode, as the working sensor, was developed allowing the successful detection and quantitative determination of DIB in serum at a concentration that can be measured in medicated patients. Thus, this optimized SPE/SW-AdSV approach might be of particular relevance as the first analytical procedure sensitive enough to determine this novel organic molecule of medical importance in real serum samples from treated patients.

Acknowledgements The study was financed from MNiSW Project ZKT/MN-3.

Open Access This article is distributed under the terms of the Creative Commons Attribution 4.0 International License (http://creativeco mmons.org/licenses/by/4.0/), which permits unrestricted use, distribution, and reproduction in any medium, provided you give appropriate credit to the original author(s) and the source, provide a link to the Creative Commons license, and indicate if changes were made.

\section{References}

1. K. Sztanke, M. Sztanke, inventors, Medical University of Lublin, assignee, Polish Patent 217682, August 28 (2014)

2. M. Sztanke, J. Rzymowska, K. Sztanke, Bioorg. Med. Chem. 21, 7465-7480 (2013)

3. D. Raghuvanshi, G. Nkepang, A. Hussain, H. Yari, V. Awasthi, J. Pharm. Anal. 7, 1-9 (2017)

4. X. Jin, F. Zhou, Y. Liu, C. Cheng, L. Yao, Y. Jia, G. Wang, J. Zhang, J. Pharm. Biomed. Anal. 158, 1-7 (2018)

5. W. Wang, Q. Cai, F. Zhou, J. Liu, X. Jin, P. Ni, M. Lu, G. Wang, J. Zhang, Redox Biol. 15, 253-265 (2018)

6. M. Korolczuk, K. Tyszczuk, Chem. Anal. 52, 1015-1024 (2007)

7. K. Tyszczuk, M. Korolczuk, Bioelectrochemistry 78, 113-117 (2010)

8. K. Tyszczuk, Electroanalysis 22, 1975-1984 (2010)

9. A. Stępniowska, M. Sztanke, T. Tuzimski, M. Korolczuk, K. Sztanke, Biosens. Bioelectron. 94, 584-588 (2017)

10. A. Economou, P.R. Fielden, Analyst 128, 205-212 (2003)

11. G. Hughes, K. Westmacott, K.C. Honeychurch, A. Crew, R.M. Pemberton, J.P. Hart, Biosensors 6, 50 (2016)

12. J. Rodríguez, G. Castaňeda, I. Lizcano, Electrochim. Acta 269, 668-675 (2018)

13. B. Mokhtari, D. Nematollahi, H. Salehzadeh, J. Mol. Liq. 264, 543-549 (2018)

14. A.A. Khorshed, M. Khairy, C.E. Banks, J. Electroanal. Chem. 824, 39-44 (2018)

15. A. Yiğit, Y. Yardım, M. Çelebi, A. Levent, Z. Şentürk, Talanta 158, 21-29 (2016)

16. P.K. Kalambate, ChR Rawool, A.K. Srivastava, Sens. Actuators B 237, 196-205 (2016)

17. S.K. Hassaninejad-Darzi, F. Shajie, Mater. Sci. Eng. C 91, 64-77 (2018)

18. I. Svancara, C. Prior, S.B. Hocevar, J. Wang, Electroanalysis 22, 1405-1420 (2010)

19. B. Nigović, B. Šimunić, S. Hocevar, Electrochim. Acta 54, 56785683 (2009)

20. I. Campestrini, O.C. de Braga, I.C. Vieira, A. Spinelli, Electrochim. Acta 55, 4970-4975 (2010)

21. C.A. de Lima, A. Spinelli, Electrochim. Acta 107, 542-548 (2013)

22. J. Wang, J.M. Lu, S.B. Hocevar, P.A.M. Farias, B. Ogorevc, Anal. Chem. 72, 3218-3222 (2000)

23. O. Hammerich, B. Speiser, Organic Electrochemistry Revised and Expanded, 5th edn. (CRC Press, Boca Raton, 2016)

24. A. Economou, Trends Anal. Chem. 24, 334-340 (2005)

25. A. Bobrowski, K. Kalcher, K. Kurowska, Electrochim. Acta 54, 7214-7221 (2009)

26. K. Tyszczuk, Sens. Actuators B 156, 899-905 (2011)

27. K. Tyszczuk-Rotko, Microsc. Microanal. 18, 531-537 (2012)

28. H.E. Zittel, F.J. Miller, Anal. Chem. 37, 200-203 (1965)

29. G.G. Gerent, C.Q. Gonçalves, P.S. da Silva, A. Spinelli, Electrochim. Acta 168, 379-385 (2015)

30. H. Sopha, S.B. Hocevar, B. Pihlar, B. Ogorevc, Electrochim. Acta 60, 274-277 (2012) 


\section{Affiliations}

\section{Anna Stępniowska' ${ }^{1}$ ( Małgorzata Sztanke ${ }^{2} \cdot$ Tomasz Tuzimski $^{3} \cdot$ Mieczysław Korolczuk $^{4} \cdot$ Krzysztof Sztanke $^{5}$}

1 Department of Biochemistry and Toxicology, University of Life Sciences in Lublin, 13 Akademicka, 20-950 Lublin, Poland

2 Chair and Department of Medical Chemistry, Medical University of Lublin, 4A Chodźki Street, 20-093 Lublin, Poland

3 Department of Physical Chemistry, Chair of Chemistry, Faculty of Pharmacy with Medical Analytics Division, Medical University of Lublin, 4A Chodźki Street, 20-093 Lublin, Poland
4 Faculty of Chemistry, Maria Curie-Skłodowska University, 3 Maria Curie-Skłodowska Sq., 20-031 Lublin, Poland

5 Laboratory of Bioorganic Synthesis and Analysis, Chair and Department of Medical Chemistry, Medical University of Lublin, 4A Chodźki Street, 20-093 Lublin, Poland 